\title{
Метод формирования приоритетного перечня автоматизируемых органов управления в системах специального назначения и его программная реализация
}

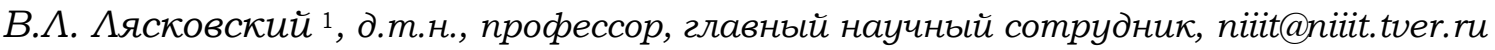

И.Б. Бреслер 1, к.в.н., доиент, генеральный директор, niiit@niiit.tver.ru

М.А. Алашеев ${ }^{1}$, к.т.н., спеииалист научно-координаиионного отдела, niiit@niiit.tver.ru

${ }^{1}$ Научно-исследовательский институт информаиионных технологий, 2. Тверь, 170100, Россия

В статье рассматриваются метод формирования приоритетного перечня органов управления распределенных информащионно-управляющих систем (РИУС) специального и военного назначения, подлежащих оснащению средствами автоматизации, а также его программная реализация в составе системы поддержки принятия решений.

Необходимость разработки и применения данного метода обусловлена тем, что создание РИУС, как правило, осуществляется в несколько этапов на протяжении длительного времени, что связано в основном с высокой трудоемкостью и стоимостью разработки, изготовления и поставки комплексов средств автоматизации, а также с ограниченными финансовыми ресурсами, технологическими и производственными возможностями всех субъектов - участников этого процесса. При этом интуитивно понятно, что оснащение одних органов управления средствами автоматизации может привносить более значимый вклад в повышение эффективности всей системы, чем автоматизация других органов управления. Однако в настоящее время отсутствует формализованный метод, позволяющий обосновать последовательность оснащения органов управления средствами автоматизации на основании учета их наиболее значимых параметров и характеристик. В связи с этим разработка метода формирования приоритетного перечня органов управления РИУС является важной практически значимой задачей.

Предлагаемый метод заключается в последовательной оценке каждого органа управления в соответствии с разработанной системой классификационных признаков. При этом все классификационные признаки иерархически взаимосвязаны, важность их убывает от первого к последнему.

Применение данного метода связано с необходимостью сбора, хранения и обработки массивов исходных данных. Для повышения удобства его использования, сокращения времени обработки информации и снижения количества ошибок, связанных с человеческим фактором, авторами осуществлена программная реализация метода как составной части разрабатываемой системы поддержки принятия решений.

Метод может быть использован заказывающими органами и научно-исследовательскими организациями при обосновании последовательности работ по созданию и развитию РИУС.

Ключевые слова: автоматизированная система, орган управления, жизненный ичикл систем управления, комплекс средств автоматизации, оснащеене средствами автоматизации.

Ряд методов и методик обоснования перечня органов и объектов автоматизации из состава распределенных информационно-управляюших систем (РИУС), подлежащих оснащению комплексами средств автоматизации (КСА), приведен в работах [1-3]. Однако в них не рассмотрены вопросы, связанные с учетом в процессе принятия решения основных значимых параметров и характеристик органов управления (ОУ), влияющих на приоритеты при оснащении средствами автоматизации.

В предлагаемом методе используется следующий подход: сначала формируется полный перечень ОУ, затем определяются приоритеты их оснащения КСА с использованием предлагаемой иерархической системы классификационных признаков. Эти приоритеты также могут быть использованы при построении поэтапной и модульной стратегии развития РИУС [4-6].

Сущность метода заключается в последовательном выполнении следующих действий.

1. Формирование перечня ОУ из состава РИУС.

Для формирования полного перечня ОУ производятся анализ руководящих документов и существующей организационной структуры 
РИУС, а также информационное обследование всех типов ОУ из состава РИУС.

В результате аналитической деятельности, выполняемой специалистами в предметной области по всем пунктам данного блока, формируется полный перечень ОУ и типов ОУ из состава РИУС.

2. Оценка приоритетов ОУ, подлежащих оснащению КСА.

Для оценки приоритетов ОУ применяется иерархия следующих классификационных признаков. $\boldsymbol{K C A}$.

Наличие КСА на ОУ, состояние и тип

В соответствии с данным признаком ОУ подразделяются на следующие группы в порядке убывания приоритета:

- орган управления не оснащен КСА;

- орган управления оснащен КСА, выработавшим ресурс (принято решение о продлении эксплуатационного ресурса);

- орган управления оснащен КСА старого парка (КСА такого типа серийно не выпускается);

- орган управления оснащен КСА нового парка.

\section{Назначение ОУ.}

По данному классификационному признаку ОУ и объекты оснащения подразделяются в зависимости от степени их влияния на достижение цели функционирования РИУС. Например, применительно к ОУ военного назначения может быть использовано разделение на следующие группы в порядке убывания приоритета:

- ОУ и объекты оснащения постоянной готовности;

- ОУ и объекты оснащения, находящиеся в войсках (штабы, командные пункты объединений, соединений и частей, включая резервные и запасные объекты);

- учебные заведения (военные учебнонаучные центры, военные академии и институты, учебные центры переподготовки специалистов).

Территориальное расположение ОУ.

По данному классификационному признаку ОУ могут быть отнесены к одной из групп в зависимости от их расположения на определенных участках территории или удаленности от каких-либо объектов.

Например, в зависимости от удаления от государственной границы ОУ подразделяются на следующие группы в порядке убывания приоритета:
- приграничные ОУ;

- ОУ, находящиеся на значительном удалении от государственной границы.

Уровень иерархии ОУ в РИУС.

Определяется структурой взаимодействия и подчиненности ОУ. Приведем пример описания иерархии ОУ применительно к системе управления военной авиацией в порядке убывания приоритета:

- главное командование;

- командование рода авиации;

- ОУ авиационных дивизий;

- ОУ авиационных полков;

- ОУ авиационных эскадрилий.

Количество подчиненных и взаимодействующих ОУ.

Определяется организационной структурой РИУС и порядком организации взаимодействия для рассматриваемого ОУ и может варьироваться в широких пределах. При этом приоритет каждого ОУ определяется порядковым номером данного ОУ в перечне, упорядоченном по убыванию количества подчиненных и взаимодействующих ОУ, в котором наименьшему порядковому номеру соответствует наивысший приоритет.

Оценка суммарной важности функциональных задач (ФЗ) на ОУ.

Для оценки приоритетов ОУ в соответствии с данным классификационным признаком каждому ОУ ставится в соответствие число, являющееся суммой важностей Ф3, реализуемых в КСА для рассматриваемого ОУ [2]. При этом, чем больше суммарная важность реализуемых задач, тем выше приоритет ОУ.

Оценка полноты и качества автоматизированного решения ФЗ на ОУ.

Оценка полноты и качества решения Ф3 характеризуется степенью их автоматизации, определяющей достигнутую эффективность автоматизированного управления с использованием КСА, находящегося на снабжении в ОУ. Для оценки могут быть использованы показатели функциональной эффективности, предложенные в [2, 7, 8].

Если для ОУ отсутствуют ФЗ, к которым предъявляются требования к вероятностныл и вероятностно-временным характеристикам (BX и BBX) их выполнения, то оценка может проводиться только с учетом их важности. В простейшем случае при одинаковой важности Ф3 оценка степени автоматизации сводится к расчету отношения количества автоматизированных Ф3 к общему количеству ФЗ. 
При одинаковом значении степени автоматизации ОУ их приоритезация может выполняться на основе следующих частных показателей:

- максимально допустимое (необходимое) количество одновременно обслуживаемых физических объектов для перспективных КСА соответствующих типов ОУ (количество одновременно обслуживаемых физических объектов определяется характеристиками, назначением и уровнем рассматриваемого ОУ);

- цикл функционирования КСА соответствующих типов ОУ (цикл функционирования КСА определяется необходимостью обеспечить требуемую реактивность РИУС по выполнению задач, решаемых в реальном масштабе времени, а также техническими характеристиками источников информации, управляемых и обслуживаемых объектов).

Остаточный ресурс эксплуатации КСА на $\boldsymbol{O У . ~}$

Процедура оценки остаточного ресурса у действующих КСА, находящихся на всех ОУ из состава РИУС для каждого планового этапа оснащения, заключается в формировании множества действующих КСА, для которых на рассматриваемом плановом этапе оснащения заканчивается срок эксплуатации. При этом предельное значение срока эксплуатации может быть задано директивно или определено на основе известных моделей [9]. Соответственно, в зависимости от остаточного ресурса КСА каждому ОУ может быть присвоен определенный приоритет - наименьшему остаточному ресурсу соответствует наивысший приоритет.

\section{Приоритет лица, принимающего реще-} нuе.

В случае неразличимости ОУ по совокупности ранее рассмотренных признаков приоритет может определяться должностным лицом, принимающим решение $[10,11]$ по формированию приоритетного перечня ОУ, подлежащих оснащению КСА.

Общая схема разработанного метода формирования приоритетного перечня ОУ, подлежащих оснащению средствами автоматизации, приведена на рисунке.

Использование предлагаемого метода предполагает необходимость сбора, хранения и обработки массивов исходных данных, объем которых зависит от количества ОУ в рассматриваемой системе управления. Очевидным путем для сокращения трудозатрат на обработку этих данных является программная реализация метода. Кроме того, это позволит минимизировать количество ошибок, связанных с вводом и обработкой информации, и повысить общее удобство использования метода. В связи с этим авторами разработана программная реализация метода как одной из составных частей системы поддержки принятия решений по созданию (развитию) РИУС.

В состав системы поддержки принятия решений входят программы:

- сбора информации о состоянии и требованиях к автоматизации РИУС;

- формирования планов по разработке КСА и оснащению ими ОУ РИУС;

- отображения планов по разработке КСА и оснащению ими ОУ РИУС;

\begin{tabular}{|c|c|c|c|c|c|}
\hline \multicolumn{6}{|c|}{ Формирование исходного перечня ОУ из состава РИУС } \\
\hline \begin{tabular}{|c|} 
Анализ руководящих \\
документов \\
\end{tabular} & \multicolumn{3}{|c|}{$\begin{array}{c}\text { Анализ существующей организационной } \\
\text { структуры РИУС }\end{array}$} & \multicolumn{2}{|c|}{$\begin{array}{l}\text { Информационное } \\
\text { обследование ОУ }\end{array}$} \\
\hline \multicolumn{6}{|c|}{ Иерархия классификационных признаков для определения приоритетов ОУ } \\
\hline $\begin{array}{l}\text { Наличие КСА на ОУ, } \\
\text { состояние и тип КСА }\end{array}$ & $\begin{array}{l}\text { Назначение } \\
\text { Оу }\end{array}$ & $\begin{array}{l}\text { Территориальное } \\
\text { расположение ОУ }\end{array}$ & $\begin{array}{r}\text { Уровє } \\
\text { иерархи } \\
\text { в РИУ }\end{array}$ & $\begin{array}{l}\text { Коли } \\
\text { и взау }\end{array}$ & $\begin{array}{l}\text { ство подчиненных } \\
\text { одействующих ОУ }\end{array}$ \\
\hline $\begin{array}{c}\text { Оценка суммарной } \\
\text { важности } \\
\text { Ф3 на ОУ } \\
\end{array}$ & \multicolumn{2}{|c|}{$\begin{array}{c}\text { Оценка полноты и качества } \\
\text { автоматизированного решения } \\
\text { ФЗ на ОУ }\end{array}$} & \multicolumn{2}{|c|}{$\begin{array}{c}\text { Остаточный ресурс } \\
\text { эксплуатации } \\
\text { КСА на ОУ } \\
\end{array}$} & $\begin{array}{c}\text { Приоритет лица, } \\
\text { принимающего } \\
\text { решение } \\
\end{array}$ \\
\hline \multicolumn{6}{|c|}{ ГВами } \\
\hline \multicolumn{6}{|c|}{$\begin{array}{c}\text { Метод формирования приоритетного перечня ОУ, подлежащих оснащееию } \\
\text { средствами автоматизации }\end{array}$} \\
\hline \multicolumn{6}{|c|}{ The method of forming a priority list of controls to be automated } \\
\hline
\end{tabular}


- сбора информации о планируемых работах по созданию КСА и оснащению ими ОУ РИУС;

- формирования приоритетного перечня ОУ РИУС, подлежащих оснащению средствами автоматизации.

На типовых экранных формах пользовательского интерфейса программной реализации предлагаемого метода представлены данные контрольного примера, соответствующие структуре, но не раскрывающие характеристики какой-либо из существующих или проектируемых РИУС.

Для ввода, редактирования и просмотра информации об организационной структуре РИУС и характеристиках входящих в ее состав ОУ предназначена экранная форма (см. http:// www.swsys.ru/uploaded/image/2019-4/2019-4dop/2.jpg). Эта информация используется в качестве исходных данных для рассматриваемого в статье метода.

На другой экранной форме (см. http://www. swsys.ru/uploaded/image/2019-4/2019-4-dop/3.

jpg) отображаются результаты применения метода формирования приоритетного перечня ОУ, подлежащих оснащению средствами автоматизации.

Табличное и схематичное отображения решений по разработке, изготовлению и продлению эксплуатации КСА на ОУ показаны на соответствующих экранных формах (см. http:// www.swsys.ru/uploaded/image/2019-4/2019-4dop/4.jpg, http://www.swsys.ru/uploaded/image/2019-4/2019-4-dop/5.jpg). При формирова- нии этих решений в качестве исходных данных используются результаты применения рассматриваемого в статье метода.

Программная реализация разработана и функционирует на основе следующего технологического стека:

- операционная система Microsoft Windows 7 и новее;

- система управления БД PostrgeSQL 9.6 и новее;

- интегрированная среда разработки Borland Delphi 7.

На составные части системы поддержки принятия решений по созданию (развитию) РИУС авторами получены Свидетельства о государственной регистрации программы для ЭВМ №№ 2018660348, 2018662688, 2018666516, 2019612091, 2019614502.

Таким образом, в настоящей статье рассмотрен метод формирования приоритетного перечня ОУ РИУС, подлежащих оснащению средствами автоматизации, применение которого позволит обосновать решения по последовательности оснащения ОУ КСА в условиях ограниченных финансовых, временных, производственных и технологических ресурсов на плановых этапах оснащения. Также в статье предложена программная реализация указанного метода как составной части системы поддержки принятия решений по созданию (развитию) РИУС, позволяющая повысить удобство использования и сократить трудозатраты, связанные с обработкой исходных данных.

\section{Лumepamypa}

1. Бреслер И.Б., Лясковский В.Л. Методика обоснования плана оснащения средствами автоматизации органов управления территориально распределенных информационно-управляющих систем организационного типа // Электронные информационные системы. 2016. № 4. С. 19-30.

2. Лясковский В.Л. Методологические основы создания (развития) и оснащения иерархических автоматизированных систем специального назначения. Тверь: Изд-во ВА ВКО, 2010. 284 с.

3. Бушуев С.Н., Осадчий А.С., Фролов В.М. Теоретические основы создания информационно-технических систем. СПб, 1998. 404 с.

4. Куприн И.Л., Давыдов А.Д., Селиванов С.Н. Модульная стратегия развития - системоэкономическая концепция интенсификации развития высокотехнологичных комплексов // Вестн. МГОУ. 2012. № 1. С. 78-85.

5. Малашенко Ю.Е., Назарова И.А. Модель управления поэтапной модернизацией гетерогенной вычислительной системы // Изв. РАН: Теория и системы управления. 2016. № 6. С. 83-96.

6. Васильев С.Н., Макаров А.А., Макаров В.Л., Махутов Н.А., Новиков Д.А. [и др.]. Управление развитием крупномасштабных систем. М.: Физматлит, 2015. 473 с.

7. Лясковский В.Л. Об одном универсальном показателе оценки функциональной эффективности иерархических автоматизированных систем организационного типа // Электронные информационные системы. 2015. № 4. С. 15-21.

8. Зацаринный А.А., Ионенков Ю.С. Некоторые аспекты оценки эффективности автоматизированных информационных систем на различных стадиях их жизненного цикла // Системы и средства информатики. 2016. Т. 26. № 3. С. 121-135. 
9. Шильникова О.В. Системный анализ и принятие решений о реинжиниринге корпоративных информационно-управляющих систем // Программные продукты и системы. 2016. № 2. C. 62-69. DOI: 10.15827/0236-235X.114.062-069.

10. Бурков В.Н., Коргин Н.А., Новиков Д.А. Введение в теорию управления организационными системами. М.: Либроком, 2009. 264 с.

11. Горелов Б.А., Давыдов А.Д., Силаев А.В., Тихонов А.В. Модели управления развитием распределенных технических систем // Изв. вузов: Машиностроение. 2018. № 3. С. 92-103. DOI: 10.18698/0536-1044-2018-3-92-103.

\section{Method of forming a priority list of automated control equipment in special purpose systems and its software implementation}

V.L.Lyaskovsky ${ }^{1}$, Dr.Sc. (Engineering), Professor, Chief Researcher,niiit@niiit.tver.ru I.B. Bresler ${ }^{1}$, Ph.D. (Military Science), Associate Professor, Director General, niiit@niiit.tver.ru M.A. Alasheev ${ }^{1}$, Ph.D. (Engineering), Professional Employee of Scientific Coordination Department, niiit@niiit.tver.ru

\section{${ }^{1}$ Research Institute of Information Technologies, Tver, 170100, Russian Federation}

Abstract. The paper considers the method of forming a priority list of control equipment for distributed information management systems (DIMS) designed for special and military applications that have to be equipped with automation tools, as well as its software implementation as a part of decision making support system.

The need to develop and apply this method arises from the fact that DIMS, as a rule, are created in several stages over a long time. This is mainly due to high complexity and cost of development, manufacture and supply of automation equipment complexes, as well as to limited financial resources, technological and production capabilities of all participants of this process. At the same time it is intuitively clear that equipping some controls with automation tools can make a more significant contribution to improving the efficiency of the entire system than automating other controls. However, there has been no formalized method till present that could substantiate the sequence of equipping controls with automation facilities based on their most significant parameters and characteristics. In this regard development of a method for forming the priority list of DIMS control equipment is a very important and practically significant task.

The essence of the proposed method lies in the consistent assessment of every unit of control equipment (CE) in accordance with the developed system of classification criteria. Moreover, all classification criteria are hierarchically interconnected. Their importance decreases from the first to the last one.

Application of the method is connected with the need to collect, store and process arrays of initial data. To use the method in a more convenient way, to reduce the time of information processing and the number of errors associated with the human factor, the authors developed the software implementing the method as an integral part of the developed decision making support system.

The method can be used by contractors and research organizations to substantiate the sequence of work in the course of DIMS development and elaboration.

Keywords: automated system, control equipment, life cycle of control systems, a set of automation tools, equipment with automation tools. 


\section{References}

1. Bresler I.B., Lyaskovsky V.L. Methods of substantiation of the plan for equipping with automation complexes for command centers of geographically distributed information management systems of organizational type. Electronic Information Systems, 2016, no. 4, pp. 19-30 (in Russ.).

2. Lyaskovsky V.L. Methodological Bases of Creation (Development) and Equipment of the Hierarchical Special Purpose Automated Systems. Tver, 2010, 284 p.

3. Bushuev S.N., Osadchiy A.S., Frolov V.M. Theoretical Basics of Information Technology Systems. St. Petersburg, 1998, p. 404 (in Russ.).

4. Kuprin I.L., Davidov A.D., Selivanov S.N. Modular development strategy - the system-economic concept of the intensification of the development of high-tech complexes. Bulletin of MRSU. 2012, no. 1, pp. 78-85 (in Russ.).

5. Malashenko Yu.E., Nazarova I.A. Management model of a phased modernization of a heterogeneous computing system. Proc. Russian Academy of Sciences. Theory and Management Systems, 2016, no. 6 , pp. 83-96 (in Russ.).

6. Vasilev S.N., Makarov A.A., Makarov V.L., Makhutov N.A., Novikov D.A (et. al.) Managing the Development of Large-Scale Systems. Moscow, Fizmatlit Publ., 2015, 473 p. (in Russ.).

7. Lyaskovsky V.L. On one universal indicator for assessing the functional efficiency of hierarchical automated systems of an organizational type. ELINS, 2015, no. 4, pp. 15-21 (in Russ.).

8. Zatsarinny A.A., Ionenkov Yu.S. Some aspects of evaluating the effectiveness of automated information systems at various stages of their life cycle. Systems and Means of Informatics. 2016, vol. 26, no. 3 , pp. 121-135 (in Russ.).

9. Shilnikova O.V. System analysis and decision-making on reengineering of corporate information management systems. Programmnye Produkty i Sistemy [Software \& Systems], 2016, no. 2, pp. $62-69$ (in Russ.) DOI: 10.15827/0236-235X.114.062-069.

10. Burkov V.N., Korgin N.A., Novikov D.A. Introduction to Organizational Management Systems Theory. Moscow, 2009, 264 p. (in Russ.).

11. Gorelov B.A., Davydov A.D., Silaev A.V., Tikhonov A.V. Models of development management of distributed technical systems. Proc. University: Engineering. 2018, no. 3, pp. 92-103 (in Russ.). DOI: 10.18698/0536-1044-2018-3-92-103.

\section{Для цитирования}

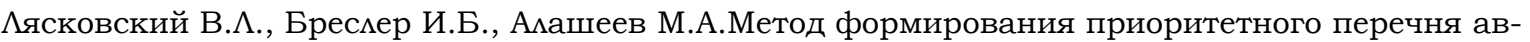
томатизируемых органов управления в системах специального назначения и его программная реализация // Программные продукты и системы. 2019. Т. 32. № 4. С. 708-713. DOI: 10.15827/0236-235X.128.708-713.

\section{For citation}

Ljaskovskij V.L., Bresler I.B., Alasheev M.A. Method of forming a priority list of automated control equipment in special purpose systems and its software implementation. Software \& Systems. 2019, vol. 32, no. 4, pp. 708-713 (in Russ.). DOI: 10.15827/0236-235X.128.708-713. 